\title{
Ankle-brachial index and the incidence of cardiovascular events in the Mediterranean low cardiovascular risk population ARTPER cohort
}

\author{
Maria Teresa Alzamora ${ }^{1,2^{*}}$, Rosa Forés ${ }^{1,2}$, Guillem Pera ${ }^{2}$, Pere Torán ${ }^{2}$, Antonio Heras ${ }^{1,2}$, Marta Sorribes ${ }^{3}$, \\ Jose Miguel Baena-Diez ${ }^{4,5}$, Magalí Urrea ${ }^{2}$, Judit Alegre ${ }^{1}$, María Viozquez ${ }^{6}$ and Carme Vela ${ }^{1}$
}

\begin{abstract}
Background: Peripheral arterial disease (PAD) of the lower limbs is a cardiovascular disease highly prevalent particularly in the asymptomatic form. Its prevalence starts to be a concern in low coronary risk countries like Spain. Few studies have analyzed the relationship between ankle-brachial index (ABI) and cardiovascular morbi-mortality in low cardiovascular risk countries like Spain where we observe significant low incidence of ischemic heart diseases together with high prevalence of cardiovascular risk factors. The objective of this study is to determine the relationship between pathological $\mathrm{ABI}$ and incidence of cardiovascular events (coronary disease, cerebrovascular disease, symptomatic aneurism of abdominal aorta, vascular surgery) and death in the $>49$ year population-based cohort in Spain (ARTPER).
\end{abstract}

Methods: Baseline ABI was measured in 3,786 randomly selected patients from 28 Primary Health Centers in Barcelona, distributed as: $\mathrm{ABI}<0.9$ peripheral arterial disease (PAD), $\mathrm{ABI} \geq 1.4$ arterial calcification (AC), $\mathrm{ABI}$ 0.9-1.4 healthy; and followed during 4 years.

Results: 3,307 subjects were included after excluding those with previous vascular events. Subjects with abnormal $\mathrm{ABI}$ were older with higher proportion of men, smokers and diabetics. 260 people presented cardiovascular events (incidence 2,117/100,000 person-years) and 124 died from any cause (incidence 978/100,000 person-years). PAD had two-fold greater risk of coronary disease (adjusted hazard ratio $(H R)=2.0,95 \%$ confidence interval (Cl) 1.3-3.2) and increased risk of vascular surgery $(H R=5.6,95 \% \mathrm{Cl} 2.8-11.5)$ and mortality $(H R=1.8,95 \% \mathrm{Cl} 1.4-2.5)$. AC increased twice risk of cerebrovascular events $(H R=1.9,95 \% \mathrm{Cl}$ 1.0-3.5) with no relationship with ischemic heart disease.

Conclusions: PAD increases coronary disease risk and AC cerebrovascular disease risk in low cardiovascular risk Mediterranean population. ABI could be a useful tool to detect patients at risk in Primary Health Care.

Keywords: Peripheral arterial disease, Ankle-brachial index, Cardiovascular diseases, Incidence, Primary health care, Cohort studies

\section{Background}

Prevention and early diagnosis of arteriosclerotic disease is one of the essential objectives in the field of cardiovascular disease since it is the main cause of mortality in developed countries. In Spain, cardiovascular diseases

\footnotetext{
* Correspondence: maiteal2007@gmail.com

'Primary Healthcare Centre Riu Nord-Riu Sud, Institut Català de la Salut, Santa Coloma de Gramenet, Spain

${ }^{2}$ Unitat de Suport a la Recerca Metropolitana Nord, Institut Universitari d'Investigació en Atenció Primària Jordi Gol (IDIAP Jordi Gol), Santa Coloma de Gramenet, Spain

Full list of author information is available at the end of the article
}

are the first cause of death, producing $32 \%$ of all deaths. Ischemic heart disease causes the greatest number of cardiovascular deaths (29\%) followed by cerebrovascular disease (25\%) [1].

Peripheral arterial disease (PAD) of the lower limbs is a cardiovascular disease highly prevalent particularly in the asymptomatic form. Its prevalence starts to be a concern in low coronary risk countries like Spain. Three different groups have studied it using the ankle-brachial index (ABI) with similar methodology. They have found PAD prevalence between $3.7 \%$ and $7.6 \%$ [2-5].

\section{Biomed Central}


It is important to note that most of the PAD cases remain undiagnosed even with the presence of intermittent claudication symptoms [6]. On the other hand arterial calcification $(\mathrm{AC})$, defined as $\mathrm{ABI} \geq 1.4$, has a prevalence of $6.2 \%$ in our country in the population $>$ 49 years of age [7].

PAD is associated to high cardiovascular risk, in both symptomatic and asymptomatic forms. Several studies have found high incidence of cardiovascular events and mortality in patients with PAD. The MESA Study, carried out in the USA, observed a hazard ratio (HR) 1.8 to develop cardiovascular morbi-mortality in patients with PAD [8]. Ankle Brachial Index Collaboration metanalysis showed that patients with PAD had in ten years between 2 and 4 times higher risk to die or to present major cardiovascular events than patients with no PAD [9].

Although the relationship between $\mathrm{AC}$ and morbimortality has been less studied it seems to have a positive association. Ankle Brachial Index Collaboration metanalysis [9] found a moderate association with HR between 0.9 and 1.5 whereas the MESA Study observed HR 1.8 to suffer a cardiovascular event or dead in patients with $\mathrm{AC}$ compared with healthy patients [8].

Few studies have analyzed the relationship between $\mathrm{ABI}$ and cardiovascular morbi-mortality in low cardiovascular risk countries like Spain where we observe significant low incidence of ischemic heart diseases together with high prevalence of cardiovascular risk factors [10-12]. Carbayo et al. obtained HR 1.7 for cardiovascular events or death in patients with PAD with no history of previous episodes [13]. Moreover, Merino et al. showed that PAD increased the risk to suffer a major coronary episode only including men in the study regardless of previous events [14].

The objective of this study is to determine the relationship between pathological ABI and incidence of cardiovascular events (coronary disease, cerebrovascular disease, symptomatic aneurism of abdominal aorta, vascular surgery) and death in the $>49$ year populationbased cohort in Spain (ARTPER) [7]. Once it is confirmed an association of PAD and/or AC to increased incidence of cardiovascular events in a low cardiovascular risk country as Spain then ABI can be recommended as a simple, fast and inexpensive tool in the Primary Health Care setting to detect patients at risk.

\section{Methods}

The ARTPER Study is an ongoing prospective observational population-based cohort study initiated in October 2006. A detailed description of the methodology of the study has been published elsewhere [15].

Briefly, at baseline ABI was measured in 3,786 randomly selected patients over the age of 49 years registered in 28 Primary Health Care centers of Barcelona region from a database including the population ascribed in the participating centers, which is even more exhaustive and updated than the census. All the subjects entered in one of the following cohorts: $\mathrm{ABI}<0.9$ peripheral arterial disease, $\mathrm{ABI} \geq 1.4$ arterial calcification, $\mathrm{ABI}$ between 0.9 and 1.4 healthy. An average of 4-year follow up was carried out, with phone contacts undertaken every 6 months from baseline to August 2012.

\section{End points}

The appearance of any of the events: myocardial infarction, angina, stroke, transient ischemic attack, symptomatic aneurysm of abdominal aorta, vascular surgery (coronary, intracranial and extracranial); and vascular and overall mortality were recorded through electronic medical records, computerized clinical history, telephone interviews with the subject or with a relative, personal or telephone interview with the general practitioner in charge of the patient, the emergency departments and emergency paramedical services, and the mortality statistical records. Finally, all the events have been checked by a medical committee whose members perform routine clinical practice.

Incidence events have been grouped as follows: coronary disease (acute myocardial infarction or angina), cerebrovascular disease (stroke or transient ischemic attack), symptomatic aneurysm of the abdominal aorta (SAAA), vascular surgery, cardiovascular morbidity (any of the 4 previous ones), mortality (vascular or non-vascular cause), overall mortality and morbid-mortality (any of the mentioned events). It was only taken into account the first episode for each type of event. Any patient that had an event at the time of recruiting or a history of an event was excluded from the analysis.

\section{Statistical analysis}

PAD and AC patient baseline profiles were separately compared to healthy patients using Chi-square test. Incidence was calculated as the number of observed events per 100,000 person-year (py), calculating Poisson 95\% confidence interval (CI). Incidence of every event was associated to each of the three cohorts by means of Cox proportional hazard models, "healthy" was the reference category. Univariate associations were investigated first, and then multivariable models were carried out, adjusting by age, gender, smoking (ever = current + former), abdominal and general obesity, hypertension, hypercholesterolemia and diabetes, calculating $\mathrm{HR}$ and 95\% CI. For each event of the study, likelihood ratio tests were used to assess the interaction of PAD (or AC) with the adjusting variables listed above. Age, abdominal and general obesity were used as continuous in Cox and interaction models. Kaplan-Meier survival function curves were done, comparing survival rates per event 
among the cohorts by log-rank test. A p-value less than 0.05 was considered statistically significant. Statistical analysis was performed with Stata 12.1 (StataCorp LP) software.

\section{Ethics}

This study was approved by the local Ethics Committee (IDIAP Jordi Gol Foundation of Investigation in Primary Care and Instituto de Salud Carlos III).

Informed written consent was obtained from all the participants. Likewise, the recommendations of the World Medical Association Declaration of Helsinki were followed.

\section{Results}

At baseline (2006-2008) 3,786>49 year old people were enrolled representing 63\% participation. Baseline prevalence of PAD and AC was $7.6 \%$ and $6.2 \%$ respectively [4]. 479 members of the ARTPER cohort previously presented some cardiovascular event and therefore were excluded from the analysis of the study. 3,307 people were enrolled to follow up, 193 (5.8\%) had PAD and 198 (6.0\%) AC. Demographics of the study population are shown in Table 1. $44 \%$ were men. Mean age at enrolment was 64.2 years (standard deviation (SD) 8.7, range 49-97). Patients with PAD or AC were older (6 and 2 years respectively) with higher proportion of men, smokers, hypertensive and diabetics. Patients with AC were more likely to be obese and patients with PAD more likely to present hypercholesterolemia.

3,307 subjects were followed up during an average of 3.83 years (SD 0.73, range 59 days- 5.56 years, median 4.03 years) adding up 12,677 person-years. 260 participants presented cardiovascular events (incidence 2,117/

Table 1 Baseline variables by ankle-brachial index (ABI)

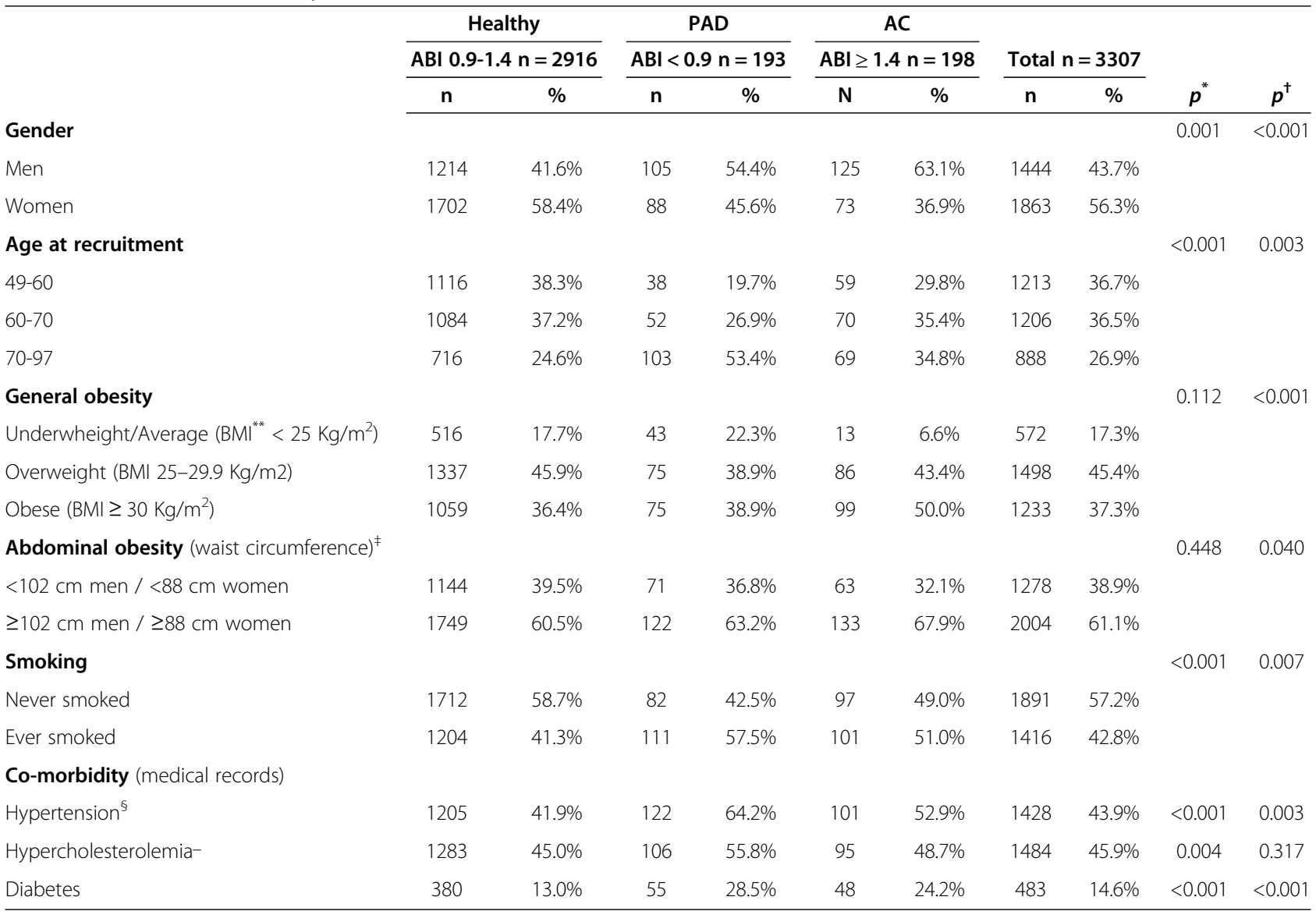

ABI: ankle-brachial index, PAD: peripheral arterial disease, AC: arterial calcification.

Individuals with prevalent events excluded.

*: $p$-value comparing $\mathrm{ABI}<0.9$ with $\mathrm{ABI}$ 0.9-1.4.

t: $p$-value comparing $\mathrm{ABI} \geq 1.4$ with $\mathrm{ABI}$ 0.9-1.4.

All comparisons made with chi-squared test.

**: BMl: body mass index.

$\neq 0,23$ and 2 missing values among each $A B I$ group.

$\S 3,41$ and 7 missing values among each $A B I$ group.

_ 3, 67 and 3 missing values among each $A B I$ group. 
100,000 py), among them 140 had coronary disease, 107 cerebrovascular disease, 19 SAAA and 45 vascular surgery. 124 patients died from any cause (incidence 978/ 100,000 py), 29 of them from vascular disease. A total of 347 patients suffered one cardiovascular event and/or died (Table 2). Kaplan-Meier survival curves are shown in Figure 1, being significant worse for PAD in coronary disease, cardiovascular morbidity and mortality and for $\mathrm{AC}$ in cerebrovascular disease.

PAD increased two- fold the risk of coronary disease $(\mathrm{HR}=2.0,95 \% \mathrm{CI} 1.3-3.2)$ and also increased the risk of vascular surgery $(\mathrm{HR}=5.6,95 \% \mathrm{CI} 2.8-11.5)$, cardiovascular morbidity $(\mathrm{HR}=2.1,95 \% \mathrm{CI} 1.5-2.9)$ and morbimortality ( $\mathrm{HR}=1.8,95 \%$ CI 1.4-2.5) irrespective of other cardiovascular risk factors. Although not statistically significant PAD also increased the risk to suffer SAAA and vascular mortality. AC increased twice the risk to present cerebrovascular events $(\mathrm{HR}=1.9,95 \% \mathrm{CI} 1.0-3.5)$ with no relationship with ischemic heart disease (Table 2).

It was observed that the effect of PAD on coronary disease was higher in men, in younger, but lower in abdominal obese, in smokers, in non-hypertensives and in diabetics. When applying interaction test this effect was only statistically significant for hypertension (Figure 2). This pattern was maintained linking PAD with cardiovascular morbidity, now with lower effects for higher body mass index. It was statistically significant the interaction of PAD with gender, hypertension and general and abdominal obese. On the other hand, PAD had a similar effect in cerebrovascular disease for each level of cardiovascular risk factors analyzed, as occurred with overall mortality (these data is not shown).

Higher effect was observed for AC regarding cerebrovascular disease in smokers, hypertensives, diabetics and abdominal (but not general) obese. However in no case the interaction of $\mathrm{AC}$ with these risk factors was statistically significant (Figure 3). AC had the same effect over coronary disease, cardiovascular morbidity and mortality across the different levels of the cardiovascular risk factors (data not shown).

High proportion of PAD patients (70\%) presented asymptomatic PAD (133 patients). 56 patients (30\%) presented intermittent claudication. 4 had no information. The interaction between PAD and intermittent claudication was not statistically significant to any of the studied events. However, the greatest difference of the PAD effect was found for cardiovascular morbidity. Compared to healthy, those with intermittent claudication had $\mathrm{HR}=2.6$, 95\% CI 1.6-4.2, and those with asymptomatic PAD had HR = 1.7, CI 95\% 1.1-2.7.

\section{Discussion}

In the low cardiovascular risk cohort ARPER it is confirmed the importance of $\mathrm{ABI}<0.9$ as an independent risk factor to have coronary disease, cardiovascular morbidity and mortality. Meanwhile $\mathrm{ABI} \geq 1.4$ is an independent risk factor to present a cerebrovascular disease.

In Spain there are no prospective studies that analyze at the same time PAD and AC as predictors of vascular events. The Albacete general population cohort [13] and the Barcelona men cohort [14] only analyzed PAD. They found similar results to our study: HR 1.9 for overall mortality and HR 3.0 for coronary disease respectively.

Our study found that patients with PAD had two-fold the risk to present a coronary event, similar results were obtained in the MESA Study (HR 1.9) [8] and in the 16 cohort ABI Collaboration metanalysis [9], (adjusted HR 2.2 for men and 2.5 for women, defining healthy as those with ABI between 1.11 and 1.40 ).

In our cohort, patients with PAD increased two- fold the risk of cardiovascular morbidity, morbi-mortality and mortality. For mortality, the higher effect was found in vascular mortality, without reaching statistical significance. ABI Collaboration metanalysis found an adjusted HR of PAD on the overall mortality 2.3 in men and 2.4 in women and greater than 3 for cardiovascular mortality [9]. The getABI Study, performed in Primary Health Care setting like the ARTPER study, obtained adjusted HR 2.0 for mortality and 1.9 for morbi-mortality in > 65 year old cohort [16].

We did not observe any link between PAD and cerebrovascular disease. Same results were found in the MESA (HR 1.6) [8], ARIC (HR 1.9) [17] and Cardiovascular Health Study (adjusted HR 1.1) [18], although these results were not statistically significant. However, other studies like the German Cohort getABI [19] and the 6-study metanalysis of Heald et al. [20] observed that PAD incremented the risk to suffer cerebrovascular disease (HR of 1.8 and 1.4 respectively). Both studies recruited older patients than ARTPER. In the Heald metanalysis, the studies that found statistically significant effect of PAD on stroke were the ones that enrolled > 70 year old patients.

We found association between PAD and vascular surgery and SAAA, the last with no statistical significance. These associations together to the effect observed on the coronary disease could explain the increased incidence of cardiovascular morbidity in patients with PAD. Although few studies have researched the association of PAD with vascular surgery or SAAA, their results are comparable to ours, like the getABI Study (adjusted HR 1.6 for coronary-artery revascularization in patients with PAD) [21].

With regards to $\mathrm{AC}$ we found an association between $\mathrm{AC}$ and cerebrovascular disease but not with other cardiovascular diseases or mortality. Likewise the MESA Study found greater effect of AC on stroke (HR 2.7) than on coronary disease (HR 2.2) [8]. ABI Collaboration 
Table 2 Incidence of cardiovascular events among different ankle-brachial index groups

\begin{tabular}{|c|c|c|c|c|c|c|c|c|c|c|c|c|c|c|c|c|c|c|c|c|c|c|c|c|}
\hline \multirow[b]{3}{*}{ Coronary disease } & \multicolumn{4}{|c|}{ Total $\mathbf{n}=3307$} & \multicolumn{4}{|c|}{$A B I 0.9-1.4 n=2916$} & \multicolumn{8}{|c|}{$A B I<0.9 n=193$} & \multicolumn{8}{|c|}{$A B I \geq 1.4 n=198$} \\
\hline & \multirow{2}{*}{$\begin{array}{c}\mathbf{n} \\
140\end{array}$} & \multirow{2}{*}{$\frac{\mathbf{I}}{1124}$} & \multicolumn{2}{|c|}{$\mathrm{Cl} 95 \%$} & \multirow{2}{*}{$\begin{array}{c}\mathbf{n} \\
105\end{array}$} & \multirow{2}{*}{$\begin{array}{c}\mathbf{I} \\
953\end{array}$} & \multicolumn{2}{|c|}{$\mathrm{Cl} 95 \%$} & \multirow{2}{*}{$\frac{\mathbf{n}}{27}$} & \multirow{2}{*}{$\frac{1}{3808}$} & \multicolumn{2}{|c|}{$\mathrm{Cl} 95 \%$} & \multirow{2}{*}{$\begin{array}{c}\mathbf{H R} \\
2.0\end{array}$} & \multicolumn{2}{|c|}{$\mathrm{Cl} 95 \%$} & \multirow{2}{*}{$\frac{p}{0.003}$} & \multirow{2}{*}{$\begin{array}{l}\mathbf{n} \\
8\end{array}$} & \multirow{2}{*}{$\frac{\mathbf{I}}{1100}$} & \multicolumn{2}{|c|}{$\mathrm{Cl} 95 \%$} & \multirow{2}{*}{$\frac{\mathbf{H R}}{0.8}$} & \multicolumn{2}{|c|}{$\mathrm{Cl} 95 \%$} & \multirow{2}{*}{$\frac{p}{0.522}$} \\
\hline & & & 946 & 1327 & & & 780 & 1154 & & & 2510 & 5541 & & 1.3 & 3.2 & & & & 475 & 2168 & & 0.4 & 1.6 & \\
\hline Cerebrovascular disease & 107 & 853 & 699 & 1031 & 80 & 723 & 573 & 899 & 14 & 1895 & 1036 & 3180 & 1.2 & 0.7 & 2.3 & 0.505 & 13 & 1783 & 949 & 3049 & 1.9 & 1.0 & 3.5 & 0.051 \\
\hline SAAA* $^{*}$ & 19 & 150 & 90 & 235 & 14 & 125 & 69 & 211 & 4 & 537 & 146 & 1374 & 3.1 & 0.9 & 9.9 & 0.063 & 1 & 135 & 3 & 754 & 0.8 & 0.1 & 6.4 & 0.857 \\
\hline Vascular surgery & 45 & 357 & 260 & 478 & 29 & 260 & 174 & 374 & 14 & 1941 & 1061 & 3257 & 5.6 & 2.8 & 11.5 & $<0.001$ & 2 & 271 & 33 & 978 & 0.9 & 0.2 & 3.6 & 0.831 \\
\hline Cardiovascular morbidity & 260 & 2117 & 1868 & 2391 & 191 & 1754 & 1514 & 2021 & 48 & 7104 & 5238 & 9418 & 2.1 & 1.5 & 2.9 & $<0.001$ & 21 & 2935 & 1817 & 4487 & 1.2 & 0.7 & 1.9 & 0.474 \\
\hline Mortality & 124 & 978 & 814 & 1166 & 95 & 849 & 687 & 1038 & 20 & 2657 & 1623 & 4104 & 1.5 & 0.9 & 2.4 & 0.141 & 9 & 1216 & 556 & 2308 & 1.0 & 0.5 & 2.0 & 0.947 \\
\hline Vascular & 29 & 229 & 153 & 329 & 19 & 170 & 102 & 265 & 8 & 1063 & 459 & 2094 & 2.4 & 1.0 & 6.0 & 0.060 & 2 & 270 & 33 & 976 & 1.2 & 0.3 & 5.1 & 0.847 \\
\hline Non vascular & 95 & 749 & 606 & 916 & 76 & 680 & 535 & 851 & 12 & 1594 & 824 & 2785 & 1.2 & 0.6 & 2.2 & 0.598 & 7 & 945 & 380 & 1948 & 1.0 & 0.5 & 2.2 & 0.947 \\
\hline Morbi-mortality & 347 & 2826 & 2536 & 3139 & 263 & 2415 & 2132 & 2726 & 59 & 8731 & 6647 & 11263 & 1.8 & 1.4 & 2.5 & $<0.001$ & 25 & 3495 & 3495 & 5159 & 1.0 & 0.7 & 1.6 & 0.838 \\
\hline
\end{tabular}

ABl: ankle-brachial index, PAD: peripheral arterial disease, AC: arterial calcification.

*: SAAA: symptomatic aneurysm of the abdominal aorta.

Individuals with prevalent events excluded.

$\mathrm{I}=$ incidence per 100,000 person-year.

C195\% $=95 \%$ confidence interval of incidence (Poisson distribution) or of hazard ratio (Cox model),

$H R=$ adjusted hazard ratio (reference ABI 0.9-1.4). HR are based on 2,799 healthy, 187 peripheral arterial disease and 189 arterial calcification subjects.

HR are adjusted by age, gender, smoking, central and general obesity, hypertension, hypercholesterolemia and diabetes.

$p=p$-value of HR different than 1 . 

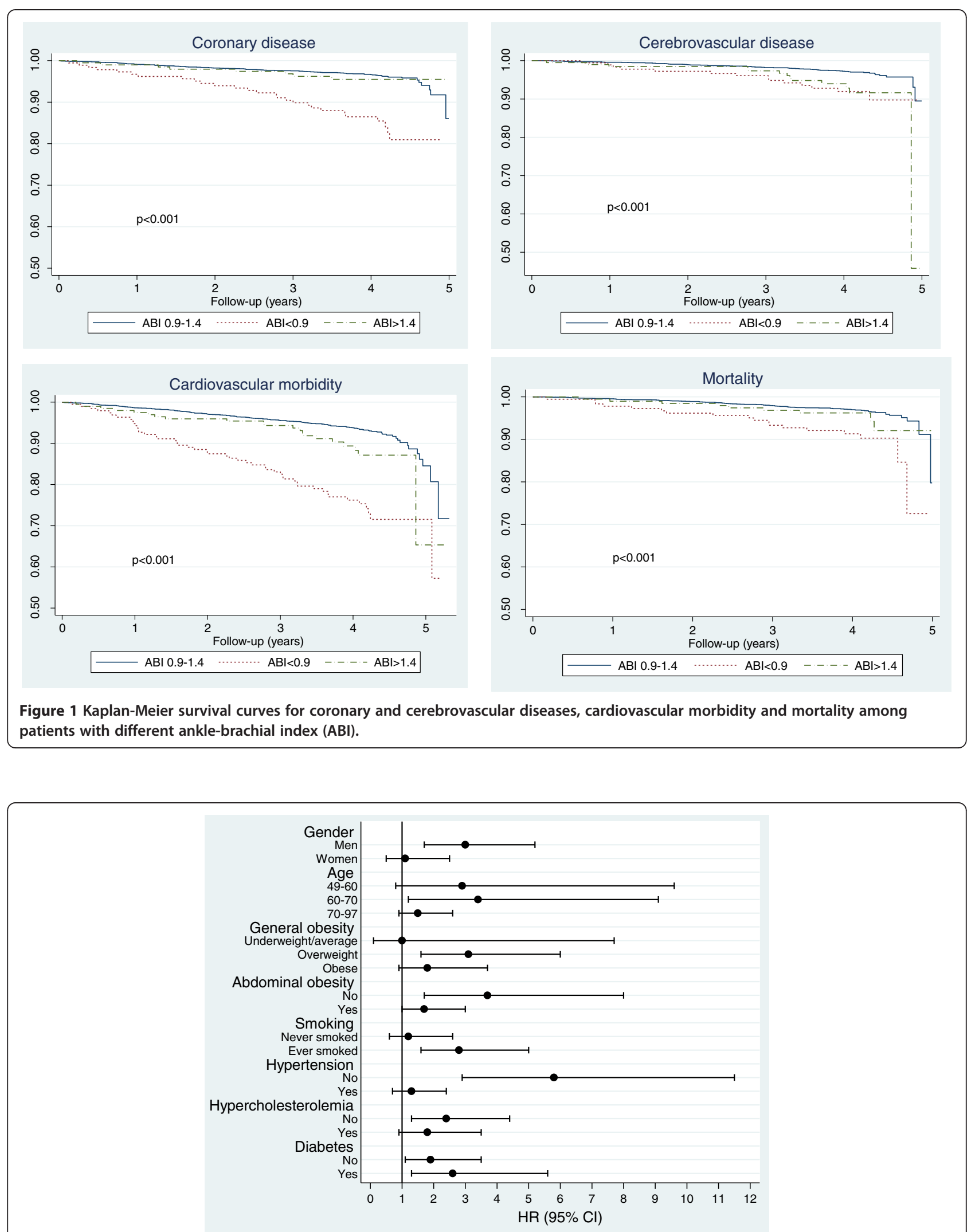

Figure 2 Effect of peripheral arterial disease for coronary disease, measured by hazard ratio (HR), among different potential cardiovascular risk factors. 


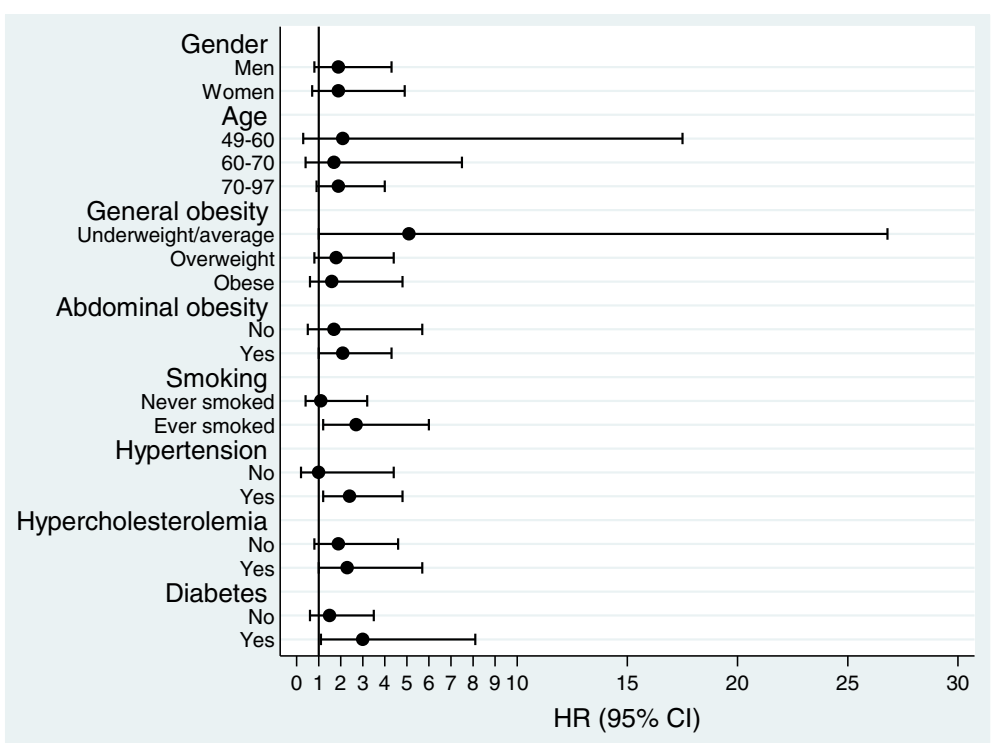

Figure 3 Effect of arterial calcification for cerebrovascular disease, measured by hazard ratio (HR), among different potential cardiovascular risk factors.

Study obtained a link between overall mortality and AC (HR 1.3) [9], but not with coronary diseases. Whereas Sutton-Tyrrell found a relationship with coronary heart disease (HR 1.5) but not with stroke or mortality in an American cohort of $>70$ year old, with AC ABI cut-off of $>1.3$ [22].

The effect of PAD was not homogeneous among the cardiovascular risk factors. PAD seems to have greater effect on coronary disease in men, in $<70$ year old patients, in overweight with no abdominal obesity, smokers, diabetes, non-hypertension and dyslipidemia. It was only statistically significant the interaction of PAD with hypertension. We obtained comparable results with PAD and cardiovascular morbidity being statistically significant the interaction with gender, age and hypertension. There is scarce information in the literature about this. In our setting it has been studied the effect of PAD on cardiovascular morbidity in diabetics with similar results (HR 2.3) [23]. Nevertheless Hansen et al. did not find any difference of the effect of PAD on mortality between diabetics and non-diabetics [24].

$70 \%$ of the patients with PAD were asymptomatic. This may delay the diagnosis of the disease and therefore have the risk to suffer a cardiovascular event in the future. However, similarly to the getABI Study [21], we obtained few differences of vascular events in patients with symptomatic PAD and asymptomatic PAD.

As described by other authors, the presence of PAD cannot be excluded in patients with AC because the arterial stiffness impedes a proper ABI measurement. Therefore, in our study the detection of patients with PAD and its effects could be underestimated [25,26]. In spite of that, both PAD and AC have shown an increase of cardiovascular risk and should be properly taken into account.

Patients with previous history of cardiovascular events were excluded from the study because they received secondary prevention treatment. Although these patients are at higher risk to get sick or to die [27] we preferred to concentrate our results in subjects to whom a primary prevention intervention should be done.

When patients with PAD were recruited we had to start secondary prevention treatment and therefore the effect of PAD on the cardiovascular events could be underestimated. Moreover, subjects may have changed their habits during the follow up period and prompted some misclassification attached to all longitudinal studies. These changes could be analyzed in future evaluations of the ARTPER cohort.

Our data is based on a 4 years follow-up, a period that could be too short to find significant associations between PAD and some of our cardiovascular outcomes or the interaction with other cardiovascular risk factors. However, despite our short follow-up we find relationship between PAD and coronary disease, vascular surgery, cardiovascular morbidity and morbi-mortality and between $\mathrm{AC}$ and cerebrovascular events, with significant interactions of PAD with gender, age and hypertension for cardiovascular morbidity.

\section{Conclusions}

In conclusion, in the low cardiovascular risk cohort ARTPER it is confirmed the importance of PAD as an independent risk factor to present coronary disease, 
cardiovascular morbidity and mortality and AC as an independent risk factor to present cerebrovascular disease. The presence or absence of risk factors can modify the effect of PAD on cardiovascular diseases and this should be further studied in the future. The measurement of PAD and AC in the Primary Health Care consultation may detect high cardiovascular risk patients. Even though $A B I$ is a simple and inexpensive instrument it will be important to define the profile of patients that are candidates to have abnormal $\mathrm{ABI}$ in order to have a more cost-effective screening tool.

\section{Abbreviations \\ ABI: Ankle-brachial index; PAD: Peripheral arterial disease; AC: Arterial calcification; HR: Hazard ratio; Cl: Confidence interval; SAAA: Symptomatic aneurysm of the abdominal aorta; py: Person-year; SD: Standard deviation.}

\section{Competing interest}

The authors declare that they have no competing interests.

\section{Authors' contributions}

MTA, RF, GP, PT, AH, MS, JMB, MU, JA, MV and CV participated in the design of the study. MTA, RF, AH, MS, JMB and MU contributed to the coordination study; GP participated in the statistical calculations. All the authors have read and approved the final manuscript.

\section{Author details}

'Primary Healthcare Centre Riu Nord-Riu Sud, Institut Català de la Salut, Santa Coloma de Gramenet, Spain. ${ }^{2}$ Unitat de Suport a la Recerca Metropolitana Nord, Institut Universitari d'Investigació en Atenció Primària Jordi Gol (IDIAP Jordi Gol), Santa Coloma de Gramenet, Spain. ${ }^{3}$ Primary Healthcare Centre Numància, Institut Català de la Salut, Barcelona, Spain. ${ }^{4}$ Primary Healthcare Centre La Marina, Institut Català de la Salut, Barcelona, Spain. ${ }^{5}$ Unitat de Suport a la Recerca de l'Àmbit de l'Atenció Primària de Barcelona Ciutat, Institut Universitari d'Investigació en Atenció Primària Jordi Gol (IDIAP Jordi Gol), Barcelona, Spain. ${ }^{6}$ Primary Healthcare Centre Singuerlín, Institut Català de la Salut, Santa Coloma de Gramenet, Spain.

Received: 2 August 2013 Accepted: 10 December 2013 Published: 17 December 2013

\section{References}

1. Defunciones Según la Causa de Muerte 2010. Instituto Nacional de Estadística. http://www.ine.es/jaxi/menu.do?type=pcaxis\&file=pcaxis\&path=\%2Ft15\% 2Fp417\%2F\%2Fa2010. Accessed March 1, 2013.

2. Ramos R, Quesada M, Solanas P, Subirana I, Sala J, Vila J, et al: Prevalence of symptomatic and asymptomatic peripheral arterial disease and the value of the ankle-brachial index to stratify cardiovascular risk. Eur I Vasc Endovasc Surg 2009, 38(3):305-311.

3. Blanes JI, Cairols MA, Marrugat J, ESTIME: Prevalence of peripheral artery disease and its associated risk factors in Spain: the ESTIME study. Int Angiol 2009, 28(1):20-25.

4. Alzamora MT, Forés R, Baena-Díez JM, Pera G, Toran P, Sorribes M, et al: The peripheral arterial disease study (PERART/ARTPER): prevalence and risk factors in the general population. BMC Public Health 2010, 10:38.5.

5. Félix-Redondo FJ, Fernández-Bergés D, Grau M, Baena Díez JM, Mostaza JM, Vila J: Prevalence and clinical characteristics of peripheral arterial disease in the study population Hermex. Rev Esp Cardiol 2012, 65(8):726-733.

6. Forés Raurell R, Alzamora Sas MT, Baena Díez JM, Pera Blanco G, Torán Monserrat P, Ingla Mas J, ARTPER group: Underdiagnosis of peripheral arterial disease in the Spanish population. ARTPER study. Med Clin (Barc) 2010, 135(7):306-309.

7. Alzamora MT, Forés R, Torán P, Pera G, Baena-Díez JM, López B, et al: Prevalence of arterial calcification and related risk factors. The multicenter population-based ARTPER study. Gac Sanit 2012, 26(1):74-77.

8. Criqui MH, McClelland RL, McDermott MM, Allison MA, Blumenthal RS, Aboyans $V$, et al: The ankle-brachial index and incident cardiovascular events in the MESA (Multi-Ethnic Study of Atherosclerosis). J Am Coll Cardiol 2010, 56(18):1506-1512.
9. Ankle Brachial Index Collaboration, Fowkes FG, Murray GD, Butcher I, Heald $\mathrm{CL}$, Lee RJ, Chambless LE: Ankle brachial index combined with Framingham Risk Score to predict cardiovascular events and mortality: a meta-analysis. JAMA 2008, 300(2):197-208.

10. Grau M, Marrugat J: Risk functions and the primary prevention of cardiovascular disease. Rev Esp Cardiol 2008, 61(4):404-416.

11. Müller-Nordhorn J, Binting S, Roll S, Willich SN: An update on regional variation in cardiovascular mortality within Europe. Eur Heart J 2008, 29(10):1316-1326.

12. Grau M, Elosua R, Cabrera de León A, Guembe MJ, Baena-Díez JM, VegaAlonso T, et al: Cardiovascular risk factors in Spain in the first decade of the 21st Century, a pooled analysis with individual data from 11 population-based studies: the DARIOS study. Rev Esp Cardiol 2011, 64(4):295-304.

13. Carbayo JA, Artigao LM, Divisón JA, Caldevilla D, Sanchís C, Torres P: Ankle brachial index and the incidence of all-cause mortality and cardiovascular morbidity in a prospective cohort study of a general population. Clin Invest Arterioscl 2011, 23(1):21-28.

14. Merino J, Planas A, De Moner A, Gasol A, Contreras C, Marrugat J, et al: The association of peripheral arterial occlusive disease with major coronary events in a Mediterranean population with low coronary heart disease incidence. Eur J Vasc Endovasc Surg 2008, 36(1):71-76.

15. Alzamora MT, Baena-Díez JM, Sorribes M, Forés $R$, Toran $P$, Vicheto $M$, et al: Peripheral Arterial Disease study (PERART): prevalence and predictive values of asymptomatic peripheral arterial occlusive disease related to cardiovascular morbidity and mortality. BMC Public Health 2007, 7:348.

16. Diehm C, Lange S, Darius H, Pittrow D, von Stritzky B, Tepohl G, et al: Association of low ankle brachial index with high mortality in primary care. Eur Heart J 2006, 27(14):1743-1749.

17. Tsai AW, Folsom AR, Rosamond WD, Jones DW: Ankle-brachial index and 7-year ischemic stroke incidence: the ARIC study. Stroke 2001, 32(8):1721-1724.

18. Newman AB, Shemanski L, Manolio TA, Cushman M, Mittelmark M, Polak JF, et al: Ankle-arm index as a predictor of cardiovascular disease and mortality in the cardiovascular health study. The cardiovascular health study group. Arterioscler Thromb Vasc Biol 1999, 19(3):538-545.

19. Meves SH, Diehm C, Berger K, Pittrow D, Trampisch HJ, Burghaus I, et al: Peripheral arterial disease as an independent predictor for excess stroke morbidity and mortality in primary-care patients: 5 -year results of the getABI study. Cerebrovasc Dis 2010, 29(6):546-554.

20. Heald CL, Fowkes FG, Murray GD, Price JF: Ankle brachial index collaboration. Risk of mortality and cardiovascular disease associated with the anklebrachial index: systematic review. Atherosclerosis 2006, 189(1):61-69.

21. Diehm C, Allenberg JR, Pittrow D, Mahn M, Tepohl G, Haberl RL, et al: Mortality and vascular morbidity in older adults with asymptomatic versus symptomatic peripheral artery disease. Circulation 2009, 120(21):2053-2061.

22. Sutton-Tyrrell K, Venkitachalam L, Kanaya AM, Boudreau R, Harris T, Thompson T, et al: Relationship of ankle blood pressures to cardiovascular events in older adults. Stroke 2008, 39(3):863-869.

23. Bundó M, Muñoz L, Pérez C, Montero JJ, Montellà N, Torán P, Pera G: Asymptomatic peripheral arterial disease in type 2 diabetes patients: a 10year follow-up study of the utility of the ankle brachial index as a prognostic marker of cardiovascular disease. Ann Vasc Surg 2010, 24(8):985-993.

24. Hanssen NM, Huijberts MS, Schalkwijk CG, Nijpels G, Dekker JM, Stehouwer CD: Associations between the ankle-brachial index and cardiovascular and all-cause mortality are similar in individuals without and with type 2 diabetes: nineteen-year follow-up of a population-based cohort study. Diabetes Care 2012, 35(8):1731-1735.

25. Suominen V, Rantanen T, Venermo M, Saarinen J, Salenius J: Prevalence and risk factors of PAD among patients with elevated ABI. Eur J Vasc Endovasc Surg 2008, 35(6):709-714.

26. Aboyans V, McClelland RL, Allison MA, McDermott MM, Blumenthal RS, Macura $\mathrm{K}$, Criqui MH: Lower extremity peripheral artery disease in the absence of traditional risk factors. The multi-ethnic study of atherosclerosis. Atherosclerosis 2011, 214(1):169-173.

27. Criqui MH, Langer RD, Fronek A, Feigelson HS, Klauber MR, McCann TJ, Browner D: Mortality over a period of 10 years in patients with peripheral arterial disease. N Engl J Med 1992, 326(6):381-386.

\section{doi:10.1186/1471-2261-13-119}

Cite this article as: Alzamora et al: Ankle-brachial index and the incidence of cardiovascular events in the Mediterranean low cardiovascular risk population- ARTPER cohort. BMC Cardiovascular Disorders 2013 13:119. 